\section{The Glomerular Fluid}

Dr. Smith discussed the analysis of the glomerular and tubular fluid obtained by Chambers's microdissection pipette by Richards, Walker, Bordley, R. C. Bradley, Bott, and Westfall. It was found that the capsular fluid was an ultrafiltrate, that glucose was reabsorbed in the proximal tubule and not the distal, and that the distal tubule was concerned with chloride reabsorption, ammonia excretion, $p \mathrm{H}$, and hypotonic urine formation.

There was lacking, however, a standard of reference to indicate the degree of water reabsorption. In 1926 Marshall and Grafflin began their studies of the aglomerular fish kidney, and showed that its blood supply was venous in nature-a counterpart of the renal portal system-that there was no glomerulus and the structure resembled the proximal tubule in man.

\section{Inulin Clearance}

By now a standard of reference was available in inulin, a polysaccharide with a large molecular weight $(5,200)$. Dr. Smith mentioned that, although the human kidney was not comparable in certain respects with that of the dog, he thought the laboratory experience with inulin was such that it must be trusted as a clinical standard. Inulin gave an identifiable clearance rate, and from the time of its introduction proper experiments began on tubular reabsorption and excretion. Shannon's study on glucose was the first to show a maximal rate in tubular transport. This maximum rate $(\mathrm{T} m)$ was subsequently determined for a number of other substances-vitamin $C$, various amino-acids, sulphates, phosphates, creatine, uric acid. Clinically this knowledge was useful, but most interesting was the implication of the possibility of complete clearance.

Dr. Smith remarked that Cushny would not have believed that the normal renal filtration rate per day was 180 litres: this contained $1,200 \mathrm{~g}$. of sodium chloride (or nearly $3 \mathrm{lb}$.), excretion being regulated to within $\pm 1 \mathrm{~g}$. a day-an indication of the importance of the kidney in relation to body fluids$400 \mathrm{~g}$. of sodium bicarbonate and 190 of glucose.

Section of the vasomotor nerves had no effect on the renal blood flow. Discussing the question of how the blood flow in the kidney is regulated, Dr. Smith remarked on the constancy of this blood flow and of the filtration rate, and suggested that the next chapter in the history of renal physiology would be headed "Glomerular-tubular balance."

\section{Sodium Excretion}

Dr. Smith then discussed sodium excretion in relation to glomerular-tubular balance. He recalled that some years ago it had been postulated that water reabsotption was carried out in two phases, obligatory and facultative, the obligatory absorption taking place in the proximal tubule and the facultative in the distal. In maximum water diuresis about $15 \%$ of the filtered water was available for excretion. In extreme osmotic diuresis $60 \%$ of water was excreted, although sodium chloride continued to be reabsorbed. It appeared that reabsorption of sodium was independent of the reabsorption of water and that it was an active process. First the sodium chloride was absorbed and then the water diffused back to maintain the osmotic pressure of the urine at approximately that of plasma.

Dr. Smith said that the thin segment consisted of very flat epithelium; many investigators thought that its function was to act as an "osmotic dialyzer." In the distal system the reabsorption of sodium chloride at least appeared to be limited by a critical constant comparable with the constants for glucose and other substances in the proximal system.

In general Merrill's theory of the genesis of oedema in chronic congestive heart failure was supported by recent studies. But it must be recognized that the tubular reabsorption of sodium was importantly influenced by adrenal cortical hormones and other factors. Finally the speaker stressed that modern methods of renal measurement were not suitable for general clinical use. The most valuable clinical method of assessment remained the urea clearance.

\section{Preparations and Appliances}

\section{TREATMENT OF FRACTURES OF TIBIA AND FIBULA}

Dr. P. R. SoNDHI, Worcester Royal Infirmary, writes : In the treatment of these fractures, with current methods of traction apparatus, it has always been a handicap to apply a plasterof-Paris cast in two stages. This usually involves handling of the leg by porters and nursing staff while the plaster is being completed. Owing to this uncertain and variable traction, angulation or even shortening can occur.

If a swivel apparatus (see Diagram) is attached to the table end of the Watson-Jones apparatus, traction can be applied with the knee held at any angle. Traction can be done with the knee at right-

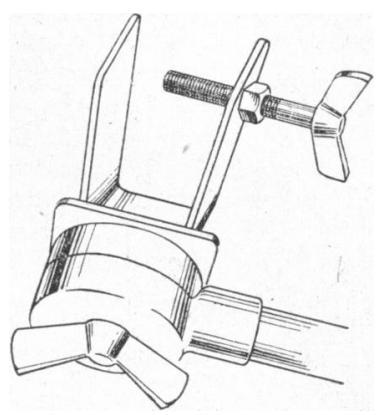
angles (Fig. 1) and the whole apparatus swung round to the position of slight flexion at the knee, when post reduction radiographs can be taken (Fig. 2). The knee is supported during this manœuvre by a sling and the hip is raised from the table by a sandbag. Cou n t e r traction is applied by raising the foot of the table. The whole leg and the fractures are thus held in a fixed position in which the cast can be applied in one stage.

Another suggested use for this apparatus is that the traction can be applied to the
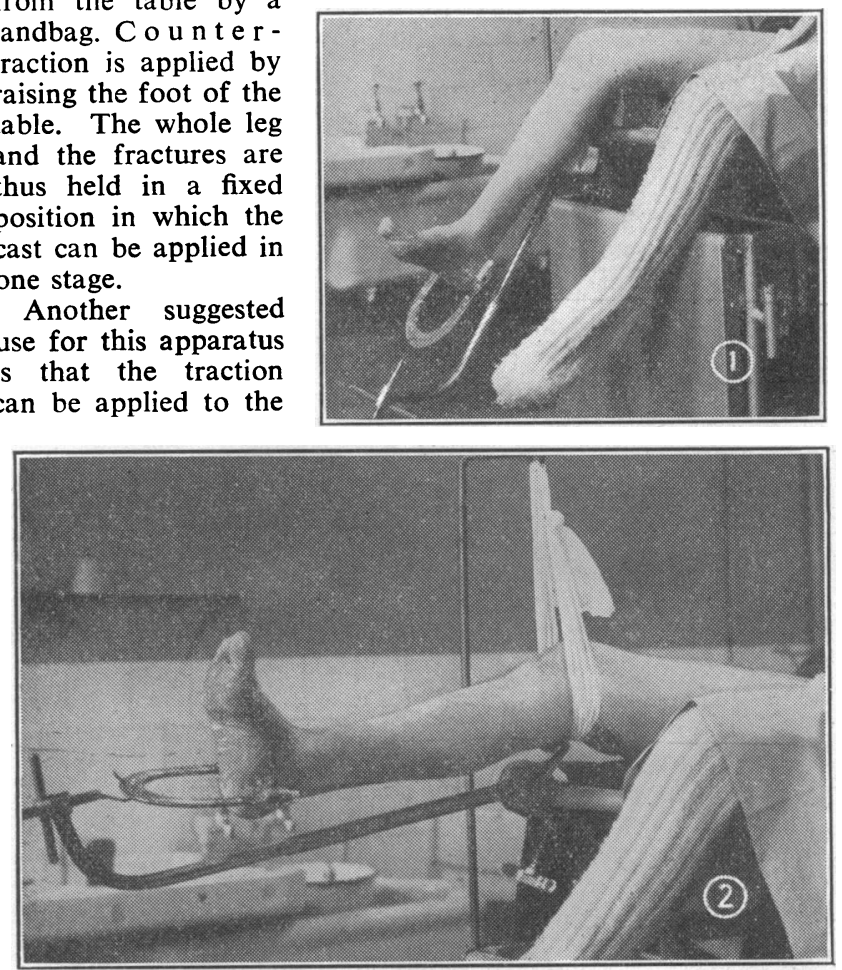

fracture with the knee in slight flexion in cases where it is not desirable or practicable to have the knee in 90 degrees flexion, such as in fracture with ankylosis of the knee. It can also be of use in compound fractures, as the leg is held in a fixed position for towelling and surgical toilet.

The Ministry of Health has sent a circular to superintendents of certified institutions saying that the Railway Executive will allow reduced fares for visitors to patients at hospitals that accommodate mainly long-stay cases. Particulars of the scheme can be obtained from the secretary of the Passenger Committee, Railway Executive, 203, Eversholt Street, London, N.W.1. 